\title{
Evaluation in vitro de l'activité antibactérienne des extrais méthanoliques des écorces de racines de Paullinia pinnata et des feuilles de Petaclethra macrophylla.
}

\author{
Lango-Yaya Ernest ${ }^{1}$, Worowounga Xavier2, Issa-Madongo Mathurin ${ }^{2}$, Namkona Armel-Frederic ${ }^{2}$, \\ Saravolia Marinette ${ }^{1}$, Rafai Donatien Clotaire ${ }^{1}$ and Syssa-Magalé Jean-Laurent ${ }^{2}$ \\ ${ }^{1}$ Laboratoire National de Biologie Clinique et de Sante Publique de Bangui, Ministère de la Sante Publique, \\ République Centrafricaine. \\ 2Laboratoire d'Architecture, d'Analyse et de Réactivité des Substances Naturelles (LAARSN), Faculté des Sciences, \\ Université de Bangui, République Centrafricaine. \\ *Auteur correspondant : worowoungax@yahoo.fr/worowoungax@gmail.com \\ Tel : (236) $72495068 / 70024051 / 77023228$
}

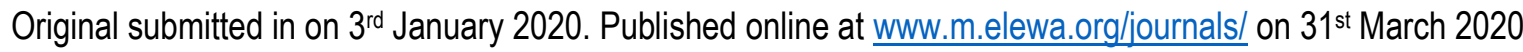
https://doi.org/10.35759/JABs.147.8

\section{RESUME}

Objectif : La résistance aux antibiotiques des souches bactériennes pose depuis quelques décennies un problème de santé publique dans le monde, car les antibiotiques de la dernière génération qui avaient un effet bactériostatique et bactéricide sur les germes deviennent de plus en plus inefficaces. C'est dans ce contexte que cette étude a été réalisée pour évaluer la sensibilité des extraits méthanolique des écorces de Paullinia pinnata et des feuilles de Petaclethra macrophylla sur les souches de Staphylococcus aureus, Shigella dysenteriae et Salmonella paratyphi $A$ en République Centrafricaine.

Méthodologie et Résultats : Pour ce travail, macération a été réalisée sous agitation pendant quatre heures pour obtenir les extraits méthanoliques de ces deux plantes. Les techniques de diffusion et de dilution ont été utilisées pour déterminer les effets de principes actifs de $P$. macrophylla et de $P$. pinnata sur les souches de $S$. aureus, de $S$. paratyphi $A$ et de $S$. dysenteriae. Le rendement de l'extraction des écorces de $P$. pinnata a été de 3,75/20 (18,76\%) et celui des feuilles de $P$. macrophylla étaient de 1,60/20 (8,00\%). Les extraits de deux plantes ont un effet bactériostatique sur la souche de $S$. aureus avec des diamètres d'inhibition respective de $25,00 \pm 0,50$ et de $27,00 \pm 0,50 \mathrm{~mm}$ ce qui n'était pas significativement différent du diamètre de la référence, le chloramphénicol $(29,00 \pm 0,00 \mathrm{~mm})$. Par contre, les extraits de $P$. pinnata et de $P$. macrophylla ont inhibé de manière sélective les deux souches $S$. dysenteriae et $S$. paratyphi $A$. Les concentrations minimales inhibitrices (CMI) des extraits varient entre 0,05 et $0,09 \mathrm{mg} / \mathrm{mL}$ et celles des concentrations minimales bactéricides (CMB) entre 0,05 et $0,14 \mathrm{mg} / \mathrm{mL}$.

Conclusion : Les deux plantes évaluées dans cette étude sont bien connues pour le traitement de quelques infections liées aux bactéries dans les différentes régions de la République Centrafricaine (RCA). Selon les résultats de cette étude, les principes actifs des extraits de ces deux plantes ont des effets bactéricides 
et bactériostatiques. II est nécessaire d'approfondir les études afin de leur exploitation et leur utilisation dans le traitement des infections bactériennes en RCA.

Mots clés : activité antibactérienne, extrait méthanolique, Paullinia pinnata, Petaclethra macrophylla

\begin{abstract}
:
Objective: Resistance of bacterial strains to antibiotics has posed a public health problem in the world in the past decades, since the last generation of antibiotic, which has a bacteriostatic and bactericidal effect, are becoming increasingly ineffective. It is in this context that this study was carried out to assess the sensitivity of the methanolic extract of Paullinia pinnata root barks and Petaclethra macrophylla leaves on Staphylococcus aureus, Shigella dysenteriae et Salmonella paratyphi A in Central African Republic.

Methodology and Results: Maceration was done under stirring for four hours to obtain the methanolic extract of the two plants; diffusion and dilution in liquid method were used to determine the effects of the active ingredients of $P$. macrophylla and $P$. pinnata on the strains of $S$. aureus, $S$. dysenteriae and $S$. paratyphi $A$. The extraction yield of $P$. pinnata root barks of $3.75 / 20(18.76 \%)$ is higher than the $P$. macrophylla leaves, which is $1.60 / 20(8.00 \%)$. The minimum inhibitory concentrations (MIC) of extracts varied between 0.05 and $0.09 \mathrm{mg} / \mathrm{mL}$ and the minimum bactericidal concentrations (MBC) was between 0.05 and $0.14 \mathrm{mg} / \mathrm{mL}$. The extracts of the two plants induced bacteriostatic activity against $S$. aureus and inhibited its growth at $25.00 \pm 0.50$ and $27.00 \pm 0.50 \mathrm{~mm}$ diameter, respectively for $P$. pinnata and $P$. macrophylla, which was not significantly different from the chloramphenicol reference at $29.00 \pm 0.00 \mathrm{~mm}$. The extracts of $P$. pinnata and $P$. macrophylla selectively inhibited $S$. dysenteriae and S. paratyphi $A$.

Conclusion: The two plants evaluated in this study are well known for their use in the treatment of bacterial infections in the different regions of Central African Republic. According to the results of this study, the active ingredients of the extracts of the two plants have bactericidal and bacteriostatic y effects on bacteria. It is necessary to conduct further studies towards their exploitation and use in treating bacterial infections in Central African Republic.
\end{abstract}

Key words: Bacterial activity, methanolic extract, Paullinia pinnata and Petraclethra macrophylla.

\section{INTRODUCTION}

Les plantes sont utilisées depuis la préhistoire par l'homme pour de besoins nutritionnels et thérapeutiques à cause de leur richesse en métabolites secondaires (Yala et al, 2016). L'utilisation des plantes médicinales en Afrique était connue par les générations passées pour longtemps (N'Guessan et al, 2011). Cependant, les métabolites secondaires contenus dans ces plantes médicinales n'étaient pas bien connus. Par ailleurs, pour lutter contre les agressions microbiennes, les chercheurs ont découvert de nombreux traitements pour soulager les patients (Etobo et al., 2017). Ces remèdes ont permis de réduire l'incidence des maladies infectieuses surtout dans les pays en voie de développement (Okombe et al., 2019). La République Centrafricaine (RCA) est située dans la zone équatoriale et possède vers le sud le prolongement de la forêt équatoriale. Le centre et le nord est couvert de la forêt arborée et de la savane herbacée. Cette végétation renferme des plantes et des herbes qui ont été utilisés et sont encore utilisés pour leur effet thérapeutique afin de traiter la plupart des maladies d'origine bactérienne, virale et parasitaire. C'est dans ce contexte que deux plantes le $P$. macrophylla et le $P$. pinnata ont été identifiées respectivement à Boukoko dans la Lobaye et à Yaloké dans l'Ombella M'poko en Republique Centrafricaine, où ils sont utilisés pour traiter certaines maladies d'origine bactérienne. Le $P$. pinnata est une liane à tige anguleuse appartenant à la famille des Sapindaceae (Annan et al, 2013) tandis que $P$. macrophylla et un arbre à la famille des Fabaceae (Souane Thirakul, 1989). En médicine traditionnelle centrafricaine diverses partie de $P$. macrophylla et $P$. pinnata sont utilisées pour traiter diverses maladies selon les informations obtenus 
lors des enquêtes ethnobotaniques. L'extrait éthanol des feuilles de $P$. pinnata récolté au Nigéria a inhibé la croissance des souches de $S$. aureus selon une étude réalisée par Olatujoye et al (2019). C'est dans ce cadre que cette étude a été réalisé d'une part pour faire le screening chimique afin de trouver les extraits de ces plantes qui contiennent les principes actifs et d'autre part

\section{MATERIEL ET METHODES}

Matériel végétale : Les feuilles de $P$. macrophylla ont été récoltées le 24 Mars 2018 à Boukoko dans la forêt et les écorces des racines de $P$. pinnata le 09 Octobre 2018 à Yaloké dans la savane en République Centrafricaine. Les herbiers, les photos des plantes et déterminer les activités antibactériennes des extraits méthanoliques des feuilles de $P$. macrophylla et les écorces des racines de $P$. pinnata. Les résultats de l'étude vont permettre d'identifier des nouvelles molécules qui contribueront pour un contrôle efficace des pathogènes bactériennes, et également de valoriser la médecine traditionnelle centrafricaine.

des organes récoltés ont été utilisés pour l'identification. Ainsi, par comparaison aux bases des données ces plantes ont été identifiées par des botanistes de la Faculté des Sciences de l'Université de Bangui, République Centrafricaine.

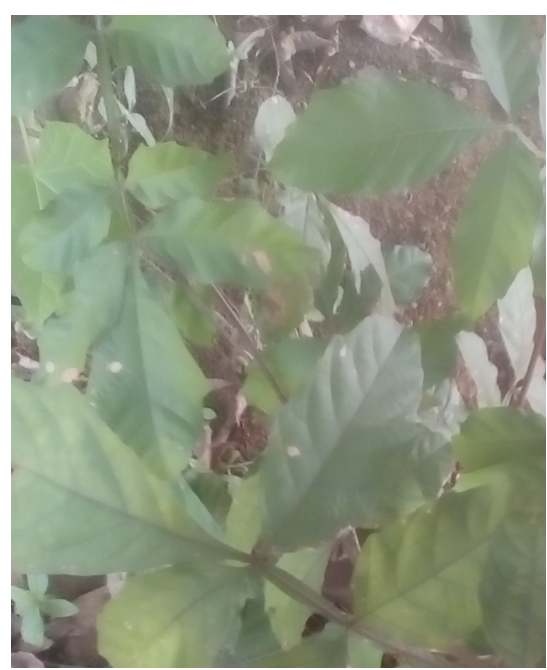

Figure 1 : les feuilles de $P$. pinnata

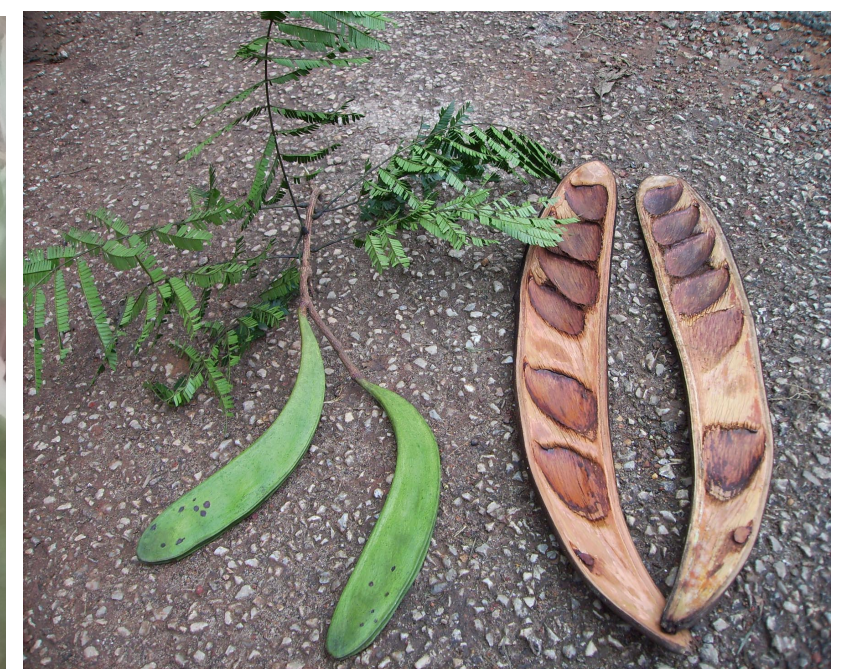

Figure 2 : les feuilles de $P$. macrophylla
Isolats bactérienne : bactériennes : Les souches de $S$. aureus ont été collectées dans les urines, celles de S. dysenterie et de S. paratyphi A dans les selles des patients venant faire des examens, par Saravolia Marinette dans le service de Bactériologie au

\section{METHODES}

Préparation de l'extrait végétal : Les échantillons des plantes prélevés étaient séchés à la température ambiante $\left(25^{\circ} \mathrm{C}\right)$ au laboratoire. Vingt gramme $(20 \mathrm{~g})$ des poudres de chaque plante étaient mélangées avec $80 \mathrm{~mL}$ de méthanol. L'ensemble a été agité pendant 4 heures sans chauffage. Après filtration, la solution a été évaporée à l'aide d'un rotavapor. Les extraits méthanoliques des deux plantes ont été utilisés pour les analyses phytochimiques et pour déterminer leurs activités biologiques.
Laboratoire National de Biologie Clinique et de Sante Publique de Bangui, Ministère de la Sante Publique, République Centrafricaine. Ces souches ont été isolées et identifiées par Dr. Lango-Yaya Ernest, Microbiologiste.

Screening phytochimique : Les méthodes standards étaient utilisées (EL-Haoud et al., 2018) avec modifications pour rechercher les familles chimiques. L'extrait a été testé qualitativement pour la présence des constituants chimiques comme les alcaloïdes, les tanins, les saponosides, les flavonoides et les steroides (Parekh et al., 2017). 


\section{Activités antibactériennes}

Teste de sensibilité de bactéries a l'extrait végétal par la méthode de diffusion: Les méthodes décrit dans la littérature (Worowounga et al., 2019 ; Okombe et al., 2019) ont été utilisées avec modification. Des disques de $4 \mathrm{~mm}$ de diamètre étaient découpés et stérilisés. Les disques stérilisés étaient imbibés dans les différentes solutions des extraits méthanoliques de $P$. macrophylla et de $P$. pinnata préparées et gardées pendant $24 \mathrm{~h}$. Les souches bactériennes isolée ont été repiquées sur les milieux Mueller Hinton par la méthode de stries et incubés à $37{ }^{\circ} \mathrm{C}$ pendant $24 \mathrm{~h}$. Deux colonies de chaque souche ont été prélevées et émulsionnées dans $5 \mathrm{~mL}$ d'eau physiologique $(\mathrm{NaCl}$ à $0,09 \%$ ) afin d'obtenir une suspension bactérienne. Cet inoculum était ensemencé par la méthode de strie sur le milieu Mueller Hinton. Les disques de $4 \mathrm{~mm}$ imprégnés de $5 \mu \mathrm{L}$ de différentes dilutions des extraits des plantes ont été placés sur le milieu Mueller Hinton. Les milieux de culture ont été ensuite incubés à l'étuve à une température de $37^{\circ} \mathrm{C}$ pendant 24 heures. Le chloramphénicol a été utilisé comme référence à des concentrations variant de 1,5 à $2,5 \mu \mathrm{g} / \mathrm{mL}$

Teste de sensibilité de bactéries a l'extrait végétal par la méthode de dilution

Préparation de l'inoculum : La méthode de Toty et al (2013) était utilisée avec modification. L'inoculum bactérien a été préparé à partir de colonies de moins de $24 \mathrm{~h}$ répliquées dans de bouillon Mueller Hinton (BMH). Un volume de suspension bactérienne de $0,1 \mathrm{~mL}$ a été prélevé respectivement pour les souches de $S$. dysenteriae et $S$. paratyphi $A$, par contre le volume pour les souches de $S$. aureus le volume était

\section{RESULTATS ET DISCUSSIONS}

L'identification des espèces végétales s'était faite sur la base des données botaniques et des noms vernaculaires attribués à chacune des plantes par les communautés locales. $P$. pinnata et nommé Kolokogo en langue Langbassi et $P$. macrophylla et connu comme Owala, mubala, gbagba, mbalanga en langue Issongo (Mbati).

Étude ethnomédicinale: Les enquêtes éthnobotaniques ont été réalisées à Yaloké et Boukoko. Quatre familles de chaque village ont été ciblées. La personne âgée de la famille qui a des connaissances sur l'utilisation de la pharmacopée traditionnelle, a été sollicitée pour l'entretien oral. Les questions ont été orientées sur les plantes médicinales utilisées dans les traitements des infections bactériennes dans le village, l'organe prélevé, les modes de préparation et de $1 \mathrm{~mL}$. Ces suspensions ont été ajoutées à $10 \mathrm{~mL}$ de $\mathrm{BMH}$ stérile dans des différents tubes. Cette suspension bactérienne réalisée était évaluée $10^{6}$ cellules/MI, et constituée d'une dilution au $10^{\circ}$ ou l'inoculum mère. La numération de l'inoculum a été réalisée selon la méthode de Worowounga et al (2019). La lecture des résultats tient compte de la turbidité de la solution pour confirmer la multiplication des cellules bactériennes. Ce qui permet de déterminer l'efficacité des extraits ou la résistance des bactéries aux différentes concentrations utilisées.

Préparation de la gamme de concentration des extraits végétaux : Trois solutions des concentrations $1,5 \mathrm{mg} / \mathrm{mL}, 1 \mathrm{mg} / \mathrm{mL}$ et $0,5 \mathrm{mg} / \mathrm{mL}$ de l'extrait méthanol de chaque plante ont été préparées

Dilution: L'inoculum bactérien a été préparé en prélevant une colonie de la culture mère, qui a été introduite dans un tube stérile contenant $10 \mathrm{~mL}$ d'eau physiologique (Yala et al., 2016). Quatre tubes à hémolyse contenant chacun un volume de $1 \mathrm{~mL}$ de la suspension bactérienne ou l'inoculum mère. Ensuite, nous avons ajouté dans les 3 premiers tubes à hémolyse, $1 \mathrm{~mL}$ d'extrait végétal des concentrations 1,$5 ; 1$ et $0,5 \mathrm{mg} / \mathrm{mL}$ respectivement. Le 4 ème tube, qui a servi de contrôle positif a reçu $1 \mathrm{~mL}$ de BMH stérile. Par conséquent, la concentration dans les tubes a été réduite de moitié du fait de la dilution volume/volume ainsi réalisée. Ces tubes ont été incubés à $37^{\circ} \mathrm{C}$ pendant $24 \mathrm{~h}$ pour la détermination de la concentration minimale inhibitrice (CMI) et celle de la concentration minimale bactéricide (CMB).

d'administration. Les résultats des enquêtes ethnobotaniques ont montré que la décoction des racines $P$. pinnata est prise contre le germe Staphylocoque à Yaloké et ces environs. Les jeunes feuilles écrasées sont utilisées contre la fracture des os. Les recherches bibliographiques ont montré que les différentes parties d'organe de $P$. pinnata sont utilisés dans la médicine traditionnelle contre la fièvre, les morsures de serpent, les fractures, la blennorragie et comme aphrodisiaque (Olatujoye et al., 2019). Les écorces de $P$. macrophylla sont utilisées dans le traitement de la diarrhée, des démangeaisons et de lotion des blessures. Les feuilles soignent la convulsion et les graines sont consommées en République Centrafricaine. 
Rendement : Les résultats (Tableau 1) ont montré que l'extrait méthanolique des racines de $P$. pinnata avait un rendement de $3,75 / 20(18,76 \%)$, supérieur à celui de $P$. macrophylla. L'extraction des feuilles de $P$. pinnata avec l'éthanol $(70 \%)$ a donné un rendement de $8.31 \%$ (Olatujoye et al., 2019) inférieur à nos résultats sur les racines de la même plante. Une étude réalisée sur les écorces des tiges de $P$. pinnata par Ouattara et al (2016) en Côte-d'Ivoire ont montré que le rendement de l'extrait méthanol $(70 \%)$ des racines de $P$. pinnata après $24 \mathrm{~h}$ de macération était de $6,62 \%$. Ce rendement est inférieur à celui que nous avons obtenu pour la même plante. Les différences observées dans les deux cas pourraient provenir des méthodes et des proportions des solvants $(70 \%$ méthanol) utilisées.

Tableau 1 : les rendements d'extraction de $P$. pinnata et de $P$. macrophylla.

\begin{tabular}{|l|c|c|c|c|}
\hline \multirow{2}{*}{ Plantes } & \multirow{2}{*}{ Extraits } & Poids des bruts & Poids d'extraits & Rendement \\
\cline { 3 - 5 } & & Gramme (g) & Gramme (g) & Pourcentage (\%) \\
\hline P. pinnata & Méthanolique & 20 & 3,75 & 18,76 \\
\hline P. macrophylla & Méthanolique & 20 & 1,60 & 8,00 \\
\hline
\end{tabular}

Screening phytochimique: Les tests de screening chimique réalisés ont permis de mettre en évidence la présence des alcaloïdes et flavonoides dans l'extrait méthanolique des deux plantes (Tableau 2). Cependant, nous avons noté la présence des tannins et saponisides uniquement dans I"extrait de P. macrophylla. Les saponisides ont été trouvés en quantité infine (Tableau 2). Les résultats des travaux de Ngono et al (2019) sur les écorces de la tige de $P$. pinnata en Côte d'lvoire ont montré la présence des flavonoïdes, l'absence des tannins et des glucosides (saponosides) dans les extraits des racines. N'guessan et al (2009) ont identifié des alcaloïdes et des flavonoïdes dans de l'extrait méthanolique des feuilles de $P$. pinnata mais pas des tannins et des saponisides dans ce même extrait. Ces résultats sont en adéquation aux résultats de notre étude pour l'extrait méthanolique des écorces des racines de cette plante. La présence des tannins et flavonoïdes dans l'extrait de $P$. macrophylla, de notre étude est en accord avec les résultats des travaux de Fungo et al (2015) sur les graines de cette plante au Cameroun. Les différences de teneur en tannins, des flavonoïdes et des glucosides dans les différentes études réalisées en Côte d'Ivoire et en RCA pourraient être dans la méthodologie de préparation et d'extraction des échantillons.

Tableau 2 : Résultats du screening chimique

\begin{tabular}{|l|c|c|}
\hline \multicolumn{1}{|c|}{ Les composés } & P. pinnata & P. macrophylla \\
\hline Alcaloïdes & $\mathbf{+ +}$ & $\mathbf{+ + +}$ \\
\hline Flavonoïdes & $\mathbf{+ + +}$ & $\mathbf{+}$ \\
\hline Tannins & - & $\mathbf{+ +}$ \\
\hline Stéroïdes & - & $\mathbf{+}$ \\
\hline Saponosides & - & $\mathbf{+}$ \\
\hline
\end{tabular}

$$
\text { Légende : +++ : Très abondant ;++ : Présence ;+ : Trace ; - : Négatif. }
$$

Sensibilité de bactéries à l'extrait végétal: Les extraits de $P$. pinnata et $P$. macrophylla ont réagi positivement sur la souche de $S$. aureus avec les diamètres d'inhibition respectivement de $25,00 \pm 0,50$ et de $27,00 \pm 0,50 \mathrm{~mm}$, comparé au chloramphénicol qui était de $29,00 \pm 0,00 \mathrm{~mm}$. L'extrait de $P$. pinnata avait un diamètre d'inhibition de $24,83 \pm 0,58 \mathrm{~mm}$ et celui de $P$. macrophylla était de $05,67 \pm 0,29 \mathrm{~mm}$ pour la souche de $S$. dysenterieae. Par conséquent l'extrait de $P$. pinnata était actif sur l'espèce de $S$. dysenteriea. Par contre, les extraits méthanoliques de ces deux plantes ont agit de manière inverse contre le $S$. paratyphi $A$ que $S$. dysenteriea (Tableau 3). Les résultats d'une étude menée par Nnennya et al (2017) au Nigéria ont montré que $10 \mathrm{mg} / \mathrm{mL}$ de l'extrait à $70 \%$ méthanol d'écorce de $P$. macrophylla ont réagi sur les souches de $S$. aureus avec une zone d'inhibition de 7,2 $\mathrm{mm}$. Bien qu'ils aient utilisé une concentration élevée de l'extrait, la zone d'inhibition est très faible que la zone d'inhibition obtenue dans notre étude (Tableau 3). Quand à Ouattara et al (2016), les résultats de leur étude menée en Côte d'Ivoire, ont donné une zone d'inhibition de 9,7 
$\mathrm{mm}$ de diamètre sur les souches de $S$. paratyphi $A$ pour une concentration de $25 \mathrm{mg} / \mathrm{mL}$ de l'extrait de méthanol de tige de $P$. pinnata. Nous avons observé que même une concentration élevée de l'extrait de $P$. pinnata n'était pas active sur les souches de $S$. paratyphi A.
Cependant, le même extrait qu'ils ont testé sur les deux souches différentes de $S$. aureus, avait des diamètres d'inhibition qui variaient de 14 à $15 \mathrm{~mm}$. Ces valeurs sont inférieures à celles que nous avons obtenus (Tableau 3).

Tableau 3 : Diamètres d'inhibition des extraits de $P$. pinnata et de $P$. macrophylla (Moyenne \pm Ecart type)

\begin{tabular}{|l|c|c|c|c|}
\hline \multicolumn{1}{|c|}{ Souches bactériennes } & \multirow{2}{*}{ Origine } & \multicolumn{1}{c|}{$\boldsymbol{P}$. pinnata } & \multicolumn{3}{c|}{ P. macrophylla } & Chloramphénicol \\
\cline { 3 - 5 } & & \multicolumn{3}{|c|}{ Diamètre d'inhibition $(\mathbf{m m})$} \\
\hline S. aureus & Urine & $25,00 \pm 0,50$ & $27,00 \pm 0,50$ & $29,00 \pm 0,00$ \\
\hline S. dysenteriae & Selles & $24,83 \pm 0,58$ & $06,67 \pm 0,29$ & $20,93 \pm 0,11$ \\
\hline S. paratyphi A & Selles & $05,67 \pm 0,29$ & $24,83 \pm 0,58$ & $22,93 \pm 0,11$ \\
\hline
\end{tabular}

Un test de kruskal wallis a été utilisé pour vérifier si les traitements diffèrent entre eux. II ya une différence significative entre les traitements (car $p$-value $=$ $0.02491(<0.05))$. Pour identifier le traitement qui diffère des autres, nous avons utilisé un test post hoc de
Nemenyi. Les résultats sont consignés dans le Tableau 4. Les résultats du test post hoc de Nemenyi montre qu'il ya une différence significative entre l'extrait de $P$. pinnata et $P$. macrophylla.

Tableau 4 : Test post hoc de Nemenyi

\begin{tabular}{|c|c|c|}
\hline & Chloramphénicol & P. pinnata \\
\hline P. pinnata & 0,37 & - \\
\hline P. macrophylla & 0,37 & 0,02 \\
\hline
\end{tabular}

Basé sur les concentrations minimales inhibitrices (CMI) et concentrations minimales bactéricides (CMB) des extraits de $P$. pinnata et de $P$. macrophylla (Tableau 5), les plantes ont un effet bactériostatique sur la souche de $S$. aureus. Elles ont réagi d'une manière similaire à la référence utilisée (Chloramphénicol). Par contre, $P$. pinnata a eu un effet bactéricide sur la souche de $S$. dysenteriae tandis que $P$. macrophylla n'a eu aucun effet. Quant à la souche de $S$. paratyphi $A$, elle a résisté à l'extrait de $P$. pinnata. Par contre l'extrait de $P$. macrophylla a réagi positivement sur la même souche avec un effet bactériostatique comparé à la référence, le chloramphénicol. Les résultats des travaux réalisés sur l'extrait méthanolique des feuilles de $P$. pinnata sur la souche de $S$. aureus par Lunga et al (2014) sont similaires à nos résultats. Les effets bactériocides et bactériostatiques observés expliquent l'utilisation de ces plantes dans le traitement des maladies d'origine bactérienne.

Tableau 5 : Paramètres antibactériens de l'extrait méthanol de $P$. pinnata et $P$. macrophylla

\begin{tabular}{|l|l|c|c|l|l|}
\hline \multicolumn{1}{|c|}{ Germes } & $\begin{array}{c}\text { Diamètre } \\
(\mathbf{m m})\end{array}$ & $\begin{array}{c}\text { CMI } \\
(\mathrm{mg} / \mathrm{mL})\end{array}$ & $\begin{array}{c}\text { CMB } \\
(\mathrm{mg} / \mathrm{mL})\end{array}$ & \multicolumn{1}{|c|}{ Extrait végétal } & Observation \\
\hline \multirow{4}{*}{ S. aureus } & $25,00 \pm 0,50$ & 0,05 & 0,09 & P. pinnata & Bactériostatique \\
\cline { 2 - 6 } & $27,00 \pm 0,50$ & 0,09 & 0,14 & $P$. macrophylla & Bactériostatique \\
\cline { 2 - 6 } & $29,00 \pm 0,00$ & $2,50(\mu \mathrm{g} / \mathrm{mL})$ & $2,50(\mu \mathrm{g} / \mathrm{mL})$ & Chloramphénicol & Bactéricide \\
\hline \multirow{5}{*}{ S. dysenteriae } & $24,83 \pm 0,58$ & 00 & 0,05 & P. pinnata & Bactéricide \\
\cline { 2 - 6 } & $06,67 \pm 0,29$ & 00 & 00 & P. macrophylla & Résistance \\
\cline { 2 - 6 } & $20,93 \pm 0,11$ & $2,00(\mu \mathrm{g} / \mathrm{mL})$ & $2,00(\mu \mathrm{g} / \mathrm{mL})$ & Chloramphénicol & Bactéricide \\
\hline S. paratyphi A & $05,67 \pm 0,29$ & 00 & 00 & P. pinnata & Résistance \\
\cline { 2 - 6 } & $24,83 \pm 0,58$ & 0,05 & 0,09 & P. macrophylla & Bactériostatique \\
\cline { 2 - 6 } & $22,93 \pm 0,11$ & $1,50(\mu \mathrm{g} / \mathrm{mL})$ & $1,50(\mu \mathrm{g} / \mathrm{mL})$ & Chloramphénicol & Bactéricide \\
\hline
\end{tabular}




\section{CONCLUSION}

Cette étude avait pour but de comprendre l'usage de $P$. pinnata et de $P$. macrophylla dans le traitement de certaines pathologies dans la population centrafricaine et de mettre en évidence les principes actifs de ces deux plantes. Ainsi les résultats de cette étude ont montré que $P$. pinnata et de $P$. macrophylla possèdent dans leurs extraits des constituants chimiques comme des alcaloïdes, des flavonoïdes et des tannins. Les activités antimicrobiennes des extraits de ces plantes ont été évaluées sur les souches des bactéries telles que les souches de $S$. aureus, $S$. dysenteriae et de $S$. paratyphi $A$. Les extraits de $P$. pinnata et de $P$. macrophylla avaient un effet bactériostatique sur les souches de $S$. aureus. L'extrait de P. macrophylla avait un effet bactériostatique sur la souche de $S$. paratyphi $A$ et l'extrait de $P$. pinnata avait un effet bactéricide sur la souche de $S$. dysenteriae. Les extraits de ces deux plantes ont agi sur les différentes souches bactériennes utilisées selon leur principe actif. Les résultats du test post hoc de Nemenyi montre qu'il ya une différence significative entre l'extrait de $P$. pinnata et $P$. macrophylla. Ces plantes sont recommandées comme pharmacopée dans le traitement de certaines infections liées aux bactéries dans différentes régions de la RCA. II est nécessaire d'envisager des moyens de formuler les extraits de ces plantes en produits à usage rationnel contre les infections bactériennes en RCA.

\section{ACKNOWLEDGMENTS}

We are very grateful to Dr SEMBOLI Olivia for its participation in the completion of this work

\section{BIBLIOGRAPHIE}

Pharmacognostic Evaluation and Physicochemical Analysis of Paullinia pinnata L. (Sapindaceae). Journal of Pharmacognosy and Phytochemistry, 2(2): 203-208.

EL-Haoud H, Boufellous M, Berrani A, Tazougart $H$ et Bengueddour R. 2018. Screening phytochimique d'une plante medicinale: Mentha spicata L. American Journal of Innovative Research and Applied Sciences, $p$ 226-233.

Etobo KJP, Oleko WR, and Nshimba SM, 2017. Activité antibactérienne des extraits bruts aqueux et concentres de quelques plantes médicinales sur les staphylocoques résistants aux antibiotiques courants à Kisangani (RD Congo). International Journal of Innovation and Scientific Research 30 (2), pp. 259-268.

Fungo R, Muyonga J, Kaaya A, Okia C, Tieguhong JC, Baidu-Forson JJ, 2015. Nutrients and bioactive compounds content of Baillonella toxisperma, Trichoscypha abut and Pentaclethra macrophylla from Cameroon. Food Science and Nutriment 3(4): 292-301.

Lunga PK, Qin XJ, Yang XW, Kuiate JR, Du ZZ, Gatsing D, 2014. Antimicrobial steroidal saponin and oleanane-type triterpenoid saponins from Paullinia pinnata. BMC Complement Altern Med. 2 (14):369.

Ngono XR, Tembe EF, Ngameni B , Nono Njinkio B, Fokunang CN, 2019. Screening
Phytochimique, Propriétés Analgésiques et Toxicité Aigüe de l'Extrait Aqueux des Écorces de la Tige de Paullinia Pinnata (Sapindaceae). Health Sciences and Diseases: 20 (6): 1-7.

N'Guessan AHO, Dago Déliko CE, Akhanovna Mamyrbékova-Békro J, Békr YA, 2011. Teneurs en composés phénoliques de 10 plantes médicinales employées dans la tradithérapie de l'hypertension artérielle, une pathologie émergente en Côte d'Ivoire. Revue de génie industriel 6: 55-61.

N'guessan K, Kadj B, Zirihi GN, Traoré D et Aké-Assi L, 2009. Screening phytochimique de quelques plantes médicinales ivoiriennes utilisées en pays Krobou (Agboville, Côte-d'Ivoire). Sciences \& Nature, 6 (1): 1-15.

Nnennaya CC, Sunday GA and Ahmadu AA, 2017. Chemical Constituents from the Stem Bark of Pentaclethra macrophylla Benth (Fabaceae). Nigerian Journal of Pharmaceutical Research, 13 (1): 37-44.

Okombe Embeya Vet Nzuzi Mavungu G, 2019. Etude de l'activité antibactérienne (in vitro) des extraits aqueux et méthanoliques de l'ail (Allium sativum L.). Journal of Applied Biosciences 141: 14419 - 14425.

Olatujoye F, Oluduro AO, Omololu-Aso J and Otusanya OO, 2019. Antibacterial Efficacy of Crude and Partially Purified Extract of Paullinia pinnata linn. on Infected Human Wound, 13: 1-11. 
Ouattara LH, Kabran GRM, Guessennd NK, Konan KF, Mamyrbekova-Bekro JA, Bekro YA, 2016. Activités antibactériennes in vitro des extraits d'écorces de racines de Mezoneuron benthamianum et de tiges de Paullinia pinnata : 2 plantes de la pharmacopée Ivoirienne. Revue CAMES - Série Pharmacopée et médecine traditionnelle africaine, 18(1) : 3140.

Parekh J, Chanda S, 2017. "In vitro Antimicrobial Activity and Phytochemical Analysis of Some Indian Medicinal Plants," Turk J Biol, 31, pp. 53-58.

Souane Thirakul S. B, 1989. Manuel de dendrologie des forêts denses. République Centrafricaine. Poulin Thériault, Québec, 525-571.

Toty AA, Guessennd N, Bahi C. Kra AM, Otokore DA et. Dosso M, (2013). Evaluation in-vitro de l'activité antibactérienne de l'extrait aqueux de l'écorce de tronc de Harungana madagascariensis sur la croissance de souches multi-résistantes, Bulletin de la Société Royale des Sciences de Liège, 82, pp. 12-21.

Worowounga X, Lango-Yaya E, Namkona AF, Boulala $\mathrm{PF}$, Saravolia M, Syssa-Magalé JL, and Kkoffi $B, 2019$. Activité antibactérienne de l'extrait méthanolique des écorces de Manilkara mabokeensis. International Journal of Innovation and Applied Studies 25 (2): 785791.

Yala JF, Ntsameso-Mve-Mba V, Azzizet Issembe Y, Lepengue NA, Souza A, 2016. Évaluation in vitro de l'activité antimicrobienne de l'extrait aqueux d'Eryngium foetidium récolté dans la ville de Franceville. Journal of Applied Biosciences $103: 9886$ - 9893. 